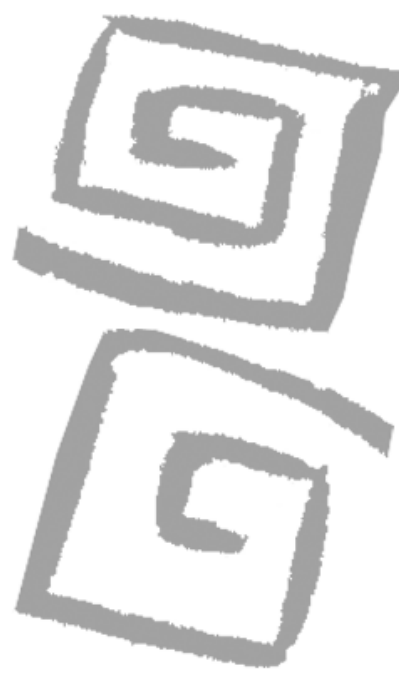

\title{
Parentesco y casas en un barrio de bajos ingresos asistido por el Programa de Salud Familiar en Salvador, Bahía, Brasil
}

\author{
Kinship and houses in a low income neighborhood \\ served by the Family Health Program in Salvador, \\ Bahia, Brazil
}

Bustamante, Vaniaํ'; McCallum, Cecilia Anne ${ }^{2}$

${ }^{1}$ Psicóloga. Doctora en Salud Colectiva. Profesora Adjunta del Instituto de Psicología, Universidade Federal de Bahia. Profesora Colaboradora del MUSA (Programa Integrado de Investigación y Cooperación Técnica en Género y Salud), Instituto de Saúde Coletiva, Universidade Federal de Bahia. vaniabus@yahoo.com

${ }^{2}$ Antropóloga. Doctora en Antropología. Profesora de la Facultad de Filosofía y Ciencias Humanas, Profesora Colaboradora del MUSA (Programa Integrado de Investigación y Cooperación Técnica en Género y Salud), Instituto de Saúde Coletiva, Universidade Federal da Bahia. cecilia.mccallum@uol.com.br
RESUMEN En este trabajo se analiza la construcción cotidiana del parentesco en un barrio de bajos ingresos de Salvador atendido por el Programa de Salud Familiar (PSF). Fueron realizadas entrevistas y observación participante durante tres años de trabajo de campo. Encontramos que el parentesco se construye permanentemente en base a vínculos de "sangre" y de "consideración". Mostramos la comprensión que los informantes tienen sobre sus "casas" y la relación entre la "configuración de las casas" y la construcción de las relaciones de parentesco de hombres y mujeres. Analizamos cómo estas relaciones son producidas en intercambios que involucran también cuidados de salud. Reflexionamos sobre los contrastes entre la propuesta formal del PSF -que establece equivalencia entre casa y familia y enfoca sobre la conyugalidad- y lo que observamos en esta investigación. A partir de eso pensamos sobre los desafíos que la implantación del mencionado programa origina, especialmente para el trabajo de los profesionales de la salud. PALABRAS CLAVE Familia; Programa de Salud Familiar; Brasil.

ABSTRACT This paper analyzes the everyday construction of kinship ties in a low income neighborhood of Salvador served by the Family Health Program (PSF). Interviews and participant observation were carried out during three years of field work. We found that kinship relations are constantly constructed on the basis of "blood ties" and conscious "consideration." We show the way informants understand the limits of their "houses" and the relationship between the "arrangement of houses" and the construction of kinship relations that include both men and women. We examine how these relationships are produced in exchanges that also involve caring for health. We reflect on the contrasts between the formal proposal of the PSF -which equates house with family and focuses on spousal relationships- and what we observe in this research. We then discuss the challenges that the implementation of the aforementioned program creates, especially for the work of health professionals.

KEY WORDS Family; Family Health Program; Brazil. 


\section{INTRODUCCIÓN}

En este artículo analizamos la construcción cotidiana de relaciones de parentesco en un barrio de bajos ingresos atendido por el Programa de Salud Familiar (PSF) en Salvador, capital del estado de Bahía, en Brasil. Articulando el análisis etnográfico con la literatura sobre cuidados de salud en familias atendidas por el PSF y con la discusión antropológica sobre familia y parentesco, buscamos contribuir a ampliar la comprensión de las "dinámicas de parentesco" (1) que permanecen poco abordadas en la literatura, y reflexionar sobre la importancia de dicha comprensión para las prácticas de salud.

EI PSF está formulado en los documentos oficiales (2-4) como parte de las acciones que buscan construir un sistema de salud afín a las directrices del Sistema Único de Salud (SUS) -universalidad, integralidad y equidad-, pensado, a su vez, como una forma de garantizar el derecho a la salud, establecido en la Constitución de 1988. Se trata de un espacio de atención básica que se propone como puerta de entrada para otros niveles de atención, teniendo como perspectiva un sistema de salud integrado.

Este programa, propuesto con el objetivo de dar cobertura a la totalidad de la población brasileña, viene presentando rápidos avances. Según datos del Ministerio de Salud, para el año 2009 se habían implementado 30.328 equipos de salud familiar, estaba presente en 5.251 municipios, y daba cobertura al $50,7 \%$ de la población, o sea, aproximadamente 96,1 millones de personas. Esto expresa el rápido aumento de la cobertura que, pocos años atrás, en 2003, era de 19.000 equipos y atendía al 37,5\% de la población (5).

EI PSF actúa a través de equipos mínimos integrados por profesionales de medicina, enfermería, auxiliares de enfermería y agentes comunitarios de salud. Los equipos ampliados también cuentan con dentistas, auxiliares de consultorio dental y técnicos en higiene dental. Cada equipo tiene como foco a la familia, la intersectorialidad del servicio y la participación activa de la comunidad, siendo responsables de acciones de prevención, promoción, recuperación, rehabilitación de los daños y de las enfermedades más frecuentes dentro de un territorio específico. Esto supone acompañar a un número de 3.000 a 4.500 personas (6). Se busca que cada equipo planifique sus acciones en función de las características de la población atendida y, al mismo tiempo, priorice algunas prácticas, en función de su importancia para la atención primaria, por ejemplo: la atención a diabéticos e hipertensos, la planificación familiar, el cuidado prenatal y la puericultura. Las actividades de los profesionales incluyen visitas domiciliarias regulares y reuniones grupales para la educación en salud.

Por ser una estrategia a partir de la cual se busca reorganizar todo el sistema de salud, el PSF ha sido objeto de numerosos estudios. Un importante tema de reflexión, que tiene fuertes implicaciones prácticas, es el concepto adoptado de familia; por lo que a continuación analizaremos algunos estudios sobre la forma en la que es tratado el concepto de familia, y también comentaremos y analizaremos algunos documentos oficiales de dicho programa.

Algunos autores incluyen las reflexiones sobre el PSF dentro de la discusión más amplia sobre la manera en que la familia es tratada en políticas sociales. Serapioni (7) señala que las atenciones brindadas a las familias son conservadoras y poco eficientes porque están sujetas a una cultura tutelar de relaciones con las clases populares, que no aceptan la autonomía de la familia. El autor también Ilama la atención sobre la fragmentación e individualización que las políticas sociales hacen de las familias, provocando duplicación o discontinuidad en la atención, que dan como resultado una serie de acciones atomizadas. Según Serapioni (7), existe consenso sobre la necesidad de retomar a la familia como unidad de atención de las políticas públicas; desarrollar redes de apoyo y compromiso en todas las familias y comunidades; y realizar una mejor integración entre familias, servicios públicos e iniciativas del sector informal.

En el contexto brasileño, la dificultad para construir prácticas de salud que enfoquen a la familia está relacionada con la existencia de múltiples formas de entenderla. Esta multiplicidad también está presente en la construcción formal del PSF. En ese sentido, Ribeiro (8), quien analizó documentos oficiales del PSF y entrevistó a gestores y profesionales de la salud, señala que incluir a la familia en los cuidados ofrecidos por 
la atención básica sobrepasa el abordaje individualizado centrado en la enfermedad, pero reconoce que no hay garantías de que esta inclusión se concrete en el PSF real, porque los diferentes actores pueden trabajar con múltiples conceptos de familia, pensando que están refiriéndose y cuidando al mismo objeto.

Los documentos sobre el PSF producidos por el Ministerio de la Salud (2-4) contienen varias presuposiciones sobre las familias. En estos documentos se afirma, como una de las cuatro atribuciones fundamentales de los profesionales de la Unidad de Salud Familiar, realizar un "abordaje integral de la familia", lo que supone un "abordaje integral de la persona, viéndola en su contexto socioeconómico y cultural con ética, compromiso y respeto" (4).

La idea de familia como "foco de la asistencia" es uno de los aspectos incluidos en el curso introductorio que es impartido a los profesionales como parte del proceso de capacitación. En este sentido, al hacer el relevamiento de las familias se registran datos demográficos, socioeconómicos y socioculturales, y esto es asociado a la "estructura familiar (composición, situación conyugal, roles, jerarquía, etc.)" (4). De esta forma, en el documento se establece una asociación entre domicilio y familia, donde a un domicilio le corresponde una familia, en la cual se espera identificar una situación conyugal. A partir de una investigación etnográfica, en este trabajo cuestionamos ese supuesto y llamamos la atención sobre la importancia de identificar las categorías construidas cotidianamente por las personas involucradas.

El proceso de trabajo de los profesionales es otro de los grandes temas de investigación vinculados al PSF. Resta y Mota (9) advierten acerca de la necesidad de reflexionar sobre las implicaciones de la propuesta del PSF, especialmente en relación con la formación de profesionales de enfermería. Los autores señalan que los profesionales necesitan construir prácticas de salud que efectivamente se dirijan a las familias, contemplando la diversidad sociocultural y comprendiendo las relaciones que la familia mantiene con vecinos y demás parientes que forman las redes de soporte social. Entre los estudios que enfocan la forma en que los profesionales perciben a las familias atendidas por el programa,
Gabardo, Junges y Selli (10) realizaron grupos focales con profesionales de diferentes categorías del PSF de un municipio del estado de Rio Grande do Sul, y encontraron que los profesionales identifican diversas organizaciones familiares. Lo que Ilama la atención en esta investigación es que la familia es definida a partir de la conyugalidad o de la co-residencia. Tal perspectiva también está presente en el estudio de Yunes, Mendes y Albuquerque (11) que analiza las percepciones y creencias de los agentes de salud sobre familias monoparentales atendidas en el PSF. Los autores consideran problemático que la mayoría de los agentes piensen que las familias "pobres" tienen escasas posibilidades de vencer las adversidades de la pobreza.

La producción antropológica sobre parentesco es muy amplia y no podemos abordarla aquí. Sin embargo, es importante dar algunas pinceladas sobre cómo se ha dado esa discusión en Brasil. El parentesco puede ser visto desde múltiples perspectivas: en relación al trabajo -desde perspectivas funcionalistas o marxistas- o en relación a los significados en una perspectiva estructuralista (12). Por otro lado, existe una amplia discusión sobre cuál sería el modelo de familia -en el sentido de un ideal presente en la conciencia colectiva y que orienta los comportamientos de un grupo-que predomina en Brasil. Varios autores cuestionan el modelo de familia nuclear burguesa (12-15), algunos argumentan que el modelo patriarcal todavía es el predominante $(12,16)$, otros conceptualizan la matrifocalidad (17-19) o la matriarcalidad (20).

Fonseca (15) sitúa su trabajo en torno a la preocupación por entender las "dinámicas de parentesco" con relación a la etnología clásica, lo que proporcionaría una arena de discusión más "abarcativa y flexible" que el modelo de familia nuclear; un ejemplo de esto son sus análisis sobre las relaciones entre parientes consanguíneos. Un aspecto, cuya importancia señala críticamente, es la tendencia de la mayor parte de los estudios a enfocar la atención sobre la conyugalidad y a buscar la matrifocalidad al investigar a grupos populares.

En el estudio de Marcelin (21) encontramos una discusión que implícitamente contempla las preocupaciones y críticas de Fonseca. El referido autor toma como punto de partida la 
casa y sus transformaciones -siguiendo una estrategia reciente en la antropología (22) - y critica lo que identifica como una tendencia a considerar que las personas de clases bajas no pueden construir el mundo simbólicamente, que tendrían los mismos valores que las clases altas y construirían ideologías para adaptarse. El autor defiende que estudiar la forma de construir y habitar las casas es central para entender la complejidad de las relaciones sociales que integran las experiencias de familia y parentesco.

\section{MÉTODO}

El análisis que presentamos aquí es parte de una investigación etnográfica titulada "O cuidado infantil em um bairro popular de Salvador: um estudo etnográfico" (23). El trabajo de campo -que incluyó observación participante y entrevistas con habitantes del barrio- fue realizado por la primera autora entre agosto de 2003 y octubre de 2006. Por este motivo en algunos momentos hacemos referencia a las notas de campo utilizando la primera persona.

El contacto inicial con los informantes del barrio se dio a través de profesionales del Programa de Salud Familiar que nos permitieron acompañarlos en visitas domiciliares. Después de algunas semanas optamos por concentrar el trabajo de campo en una zona de fácil acceso y cercana al puesto de salud. Para elegir a las familias que visitaríamos regularmente realizamos un relevamiento sobre la forma en que los habitantes de esa área organizaban su convivencia familiar. Así identificamos algunas formas de vivir en familia que eran las más comunes: una pareja con hijos, una madre viviendo con sus hijos, parejas con hijos conviviendo con abuelos y otros parientes. Esto nos ayudó a establecer los criterios para elegir las siete unidades residenciales que visitamos durante la mayor parte del tiempo que duró el trabajo de campo. Otros criterios fueron la facilidad para el acceso y la mutua afinidad. Al iniciar el contacto solicitamos que nos fuera permitido visitar a "la familia", lo que incluía a hombres y mujeres. De esta manera, si bien los datos que presentamos en este trabajo son principalmente provenientes del contacto con mujeres, también establecimos importantes vínculos con informantes hombres.

Como Wolf, citado por Jackson (24), consideramos que la etnografía es un trabajo realista, motivado "...por una sensación urgente de registrar y testimoniar experiencias humanas que 'nos hablan', sin superficialidad, sobre cosas que importan" (traducción nuestra). De esa forma, consideramos que la etnografía es mucho más que una forma de describir, es la mejor forma de entender y mostrar cómo viven y cómo se relacionan personas de diferentes grupos.

Para Toren (25) la observación participante es el método más característico del enfoque etnográfico. Este abordaje implica ser, al mismo tiempo, un participante y un observador que cuestiona su propia participación y la de los demás en eventos cotidianos, de manera que nada de lo que se dice es considerado irrelevante. Según esta autora, no se pretende que el análisis etnográfico esté basado en muestras representativas. En contraposición, el desafío es saber lo máximo posible sobre las personas cuyas ideas y comportamientos son objeto de análisis y, para eso, es importante hacer entrevistas en profundidad con algunos informantes.

El análisis se dio en todos los momentos de la investigación y caminó junto con el proceso de escribir (26). Las entrevistas y las notas de campo fueron transcritas, leídas y organizadas en archivos siguiendo una secuencia temporal. Las primeras lecturas del material fueron generales, teniendo como objetivo pensar sobre el problema de investigación, identificando puntos importantes. Un segundo tipo de lectura implicó la identificación de temas importantes, la selección de fragmentos relacionados y la creación de nuevos archivos. Nuevas lecturas del material seleccionado $-y$ a veces el retorno al material original- se fueron dando conforme construíamos los argumentos del estudio. En ese proceso, se fueron construyendo perspectivas más profundas sobre el mismo material. Algunos resultados importantes se dieron después de nuevas lecturas de las mismas notas.

Los resultados están organizados de la siguiente manera: inicialmente, describimos los vínculos y las actividades cotidianas que incluyen a parientes que viven en casas que integran una configuración de casas; en un segundo 
momento, analizamos la relación con "vecinosfamilia" que se da en una configuración de casas más amplia; finalmente, describimos lazos de sangre y consideración y los sentidos que estos adquieren cotidianamente. Elegimos las situaciones familiares que se destacaron por su frecuencia y, al mismo tiempo, las que mejor ilustran los aspectos relacionales que constituyen el foco de nuestra reflexión.

El protocolo de investigación (CAAE: 0025.0.069.000-06) fue aprobado por el Comité de Ética en Investigación del Instituto de Saúde Coletiva de la Universidade Federal da Bahia (ISC-UFBa). Los cuidados éticos incluyen el uso de nombres ficticios.

\section{RESULTADOS Y DISCUSIÓN}

\section{Casa y configuración de casas}

El barrio investigado -al que denominamos Prainha (a)- tiene aspectos en común con otros barrios populares: servicios insuficientes, infraestructura urbana precaria, algunas calles sin asfalto, falta de espacios verdes y de esparcimiento, presencia de casas inacabadas, entre otras. Sus habitantes tienen bajos niveles de escolaridad y de ingresos. Alternan períodos de empleo y desempleo. Los relatos sobre episodios de violencia son frecuentes, incluyendo violencia doméstica, discusiones entre vecinos y violencia practicada por la policía. Por otro lado, existe una presencia creciente de instituciones -unidades de salud de la familia, escuelas, guarderías, comisarías-y de programas sociales.

El modelo de organización domiciliario que tiene más prestigio en el barrio investigado es el de padres e hijos conviviendo en la misma casa, donde el hombre es el proveedor. Un día, Mila -una niña de 13 años- comparó dos formas de organización residencial: "normal" y "Ilena". Refiriéndose a una amiga de la misma edad sentada a su lado, decía:

"En su casa hay muchos parientes, su casa es una casa llena. Todos viven ahí. Vive la mamá, el tío, la tía, la abuela, el primo. En su casa viven un montón de parientes". Entonces yo pregunté: "¿Y tu casa cómo es?". Mila respondió que su casa es normal. Le pregunté: "¿Cómo normal?". Ella respondió: "en mi casa viven mi papá, mi mamá, mi hermana y yo". Contó que tenía dos hermanos y uno de ellos, un chico de 15 años, vive con un hombre del trabajo. Ese joven había sido entregado a otra persona, porque los padres no tenían cómo criarlo. (Notas de campo)

En este relato, Mila explica que su casa es "normal" porque está habitada apenas por ella, los padres y la hermana. Y ella considera que "normal" es mejor que "llena", como describe a la casa de la amiga. Hay un hecho que si bien fue mencionado por Mila, no fue considerado por ella como importante: su hermano está viviendo en otra casa, pero eso no afecta la "normalidad" de la casa. Esto muestra que "casa" es una categoría nativa que no se refiere solo a la construcción física, sino al grupo de personas que vive en ella y las relaciones entre ellos. La historia de doña Sonia y la organización de su cotidiano son ilustrativas de cómo se construyen lazos de parentesco en el barrio.

Cuando le pregunté a doña Sonia -en esa época de 66 años de edad-quiénes eran de su familia ella me dijo: "Yo vengo de los Oliveira de Maragogipe" (b). Mencionó el nombre de la madre, todavía viva, el del padre y el de otros parientes ya fallecidos. Cuando le pregunté si tenía parientes en el barrio, se refirió a los tres hijos menores y a los vecinos: "Mis vecinos son de mi familia", y en seguida especificó, dejando en claro que no se trataba de todos los vecinos: "Están Rosa, Augusta, que son gente especial, tienen 72 y 85 años. Es lo mismo que ser una madre. Está Aurelina, una persona muy especial. Actualmente ella es evangélica, tiene seis hijitos muy especiales".

Doña Sonia y muchas otras personas que eran vecinas, cuando vivían en habitaciones construidas sobre estacas recibieron pequeñas casas de madera en Prainha a comienzos de la década de 1980. Las transformaciones en la vivienda de doña Sonia, que dieron origen a las casas de los hijos, se han dado de una forma que es muy común en el barrio. En 2003 la casa ya era de "material noble" y continuaba en construcción. Y fue Rodrigo, el hijo preferido, quien construyó la casa formada por cinco ambientes: dos 
dormitorios, sala, cocina y baño. Doña Sonia vivía en esa casa con su hija Jeane; en la parte de arriba Rodrigo estaba construyendo una casa para vivir con su esposa y su hijo; y al lado, su hija Jussiara construyó una casa de apenas dos ambientes y vivía con su marido Cristóbal.

"Anda a la casa de mi nuera, anda a conocer a mi nieto", propuso doña Sonia en una de mis primeras visitas. Entonces decidí ir. Subí las escaleras y toqué la puerta. Lucia ya sabía que yo estaba por ahí, así como se saben muchas otras cosas que suceden cotidianamente. Ella estaba con Rodrigo y su hijo, Emerson. En el cuarto había una cama, una cuna, un armario, un televisor, algunos objetos de cocina, juguetes y otros objetos de menor tamaño. Entre otras cosas, Lucia explicó que Rodrigo estaba construyendo la casa de la pareja. Ella había dejado la casa de su madre hacía algunos meses, cuando Rodrigo terminó de construir el cuarto, porque ellos ya tenían un hijo.

Lucia tenía independencia, a pesar de que usaba la cocina y el sanitario de la casa de su suegra. Un día, algunas semanas después del inicio de mis visitas, vi un equipo de sonido en la casa, lo comenté y Lucia me dijo: "es mejor no tener que bajar para escuchar música". Ella intentaba realizar la mayor cantidad posible de actividades en su propia casa. Sin embargo, muchas veces la encontré cocinando, comiendo, dándole comida a su hijo o lavando ropa en la casa de su suegra.

La casa de Jussiara y Cristóbal, a pesar de que tenía dos ambientes, no era muy diferente a la de Lucia. Una parte de la casa de doña Rosa había sido cerrada, y se había abierto una puerta a la calle. Esta casa, formada por una sala y un cuarto, se encontraba ubicada en medio de las de doña Sonia y doña Rosa, en coincidencia con el hecho de que Cristóbal es hijo de doña Rosa, y Jussiara, de doña Sonia. El baño y la cocina de las respectivas casas maternas eran usados cotidianamente por la pareja y la pequeña Anita.

Con base en la observación del cotidiano de doña Sonia, Lucia, Jussiara y otras personas en Prainha, argumentamos que las tres viven en "casas" diferentes, a pesar de que, desde una perspectiva descriptiva, se podría decir que el lugar donde Lucia vive es solo una habitación y el de Jussiara son solo dos habitaciones, las que no constituirían casas ya que no incluyen una cocina propia. Consideramos que este criterio sugerido por Pina Cabral (27) para identificar la existencia de una casa -a partir de su investigación en el Alto Mino, en Portugal-, no es válido para aplicar en Prainha. En el contexto investigado, lo que transforma un lugar en una casa es principalmente la experiencia vivencial, el hecho de sentir que determinado espacio le pertenece a uno. En términos prácticos, la casa se refiere a un lugar donde un grupo mínimo y estable de personas -frecuentemente padres e hijos- puede dormir y guardar sus pertenencias. Se trata también de un espacio para administrar, pero no necesariamente realizar, las actividades necesarias para la supervivencia, tales como alimentación o higiene.

Cuando esas personas dicen "casa", se refieren a algo que trasciende el espacio material pero que no prescinde de él. Es necesario que exista un espacio material para poder considerar que existe una casa. Según Marcelin (21) "en 'casa', como en 'familia', existe un significado ontológico. Se trata de un lugar en el cual y por el cual cada uno se define y a partir del cual sostiene su existencia social como persona" (traducción de las autoras). Este argumento ayuda a comprender por qué, cuando los habitantes de Prainha dicen "mi casa", no se refieren necesariamente a una casa en el sentido funcional -con cocina, sanitario y otras habitaciones- sino a un espacio físico que ellos consideran como tal.

En su investigación en barrios de bajos ingresos en una ciudad del interior del estado de Bahía, Marcelin (21) identificó que "la casa solo existe en el contexto de una red de unidades domésticas. Esta es pensada y vivida en interrelación con las otras casas que participan de su construcción, en el sentido simbólico y concreto" (traducción de las autoras). La casa integra una configuración que no se refiere a un conjunto inmediatamente localizable para el investigador. No corresponde al concepto de "familia extensa". Se trata de una conceptualización, por medio de la categoría cultural "casa", de procesos relacionales entre agentes familiares originales de varias casas. Los agentes investigados se mueven en una configuración formada entre dos a siete casas que están localizadas en un espacio cercano (el barrio), menos cercano (la ciudad) y distante (la periferia de Salvador, Camaçari, etc.). 
En la configuración de casas de doña Sonia y sus hijos, varias actividades son colectivizadas: en la casa de doña Sonia se prepara la comida para los miembros de las tres casas, y a veces traen comida de otra casa. Doña Sonia lava su propia ropa y la de sus hijos y nietos. El carácter colectivo de algunas actividades contribuye a dar la impresión de límites confusos entre las casas y las personas. Poco a poco fui percibiendo algunos de los criterios existentes y la forma en que estos son negociados permanentemente. En este sentido, al comparar la casa de doña Sonia y la de sus hijos, encontramos diferencias que indican jerarquía. La primera está mejor equipada: tiene más muebles, televisor, equipo de sonido, teléfono, sanitario, heladera. Cabe a los hijos proveer muebles, ropa y alimentos. El respeto a esta jerarquía hacía que antes de visitar la casa de Lucia o la de Jussiara la investigadora tuviera que pasar primero por la casa de doña Sonia, porque además podría encontrar a alguna de ellas en este lugar, algo que raramente sucedía con doña Sonia, que no tenía la costumbre de pasar el tiempo en otras casas.

\section{"Vecinos-familia"}

Las casas de las cuatro mujeres que son "vecinas-familia" -junto con las de sus respectivos hijos- forman parte de una configuración más amplia. La Figura 1 ilustra la ubicación de las casas y al mismo tiempo su organización jerárquica.

Los intercambios entre las cuatro mujeres -doña Sonia, doña Aurelina, doña Rosa y doña Augusta- y los respectivos parientes consanguíneos, son cotidianos. Se trata de nueve casas en las que, al mismo tiempo, cada una está primariamente vinculada a un subconjunto dentro de la configuración. Por ejemplo, los miembros de las

Figura 1. Los vecinos-familia de doña Sonia

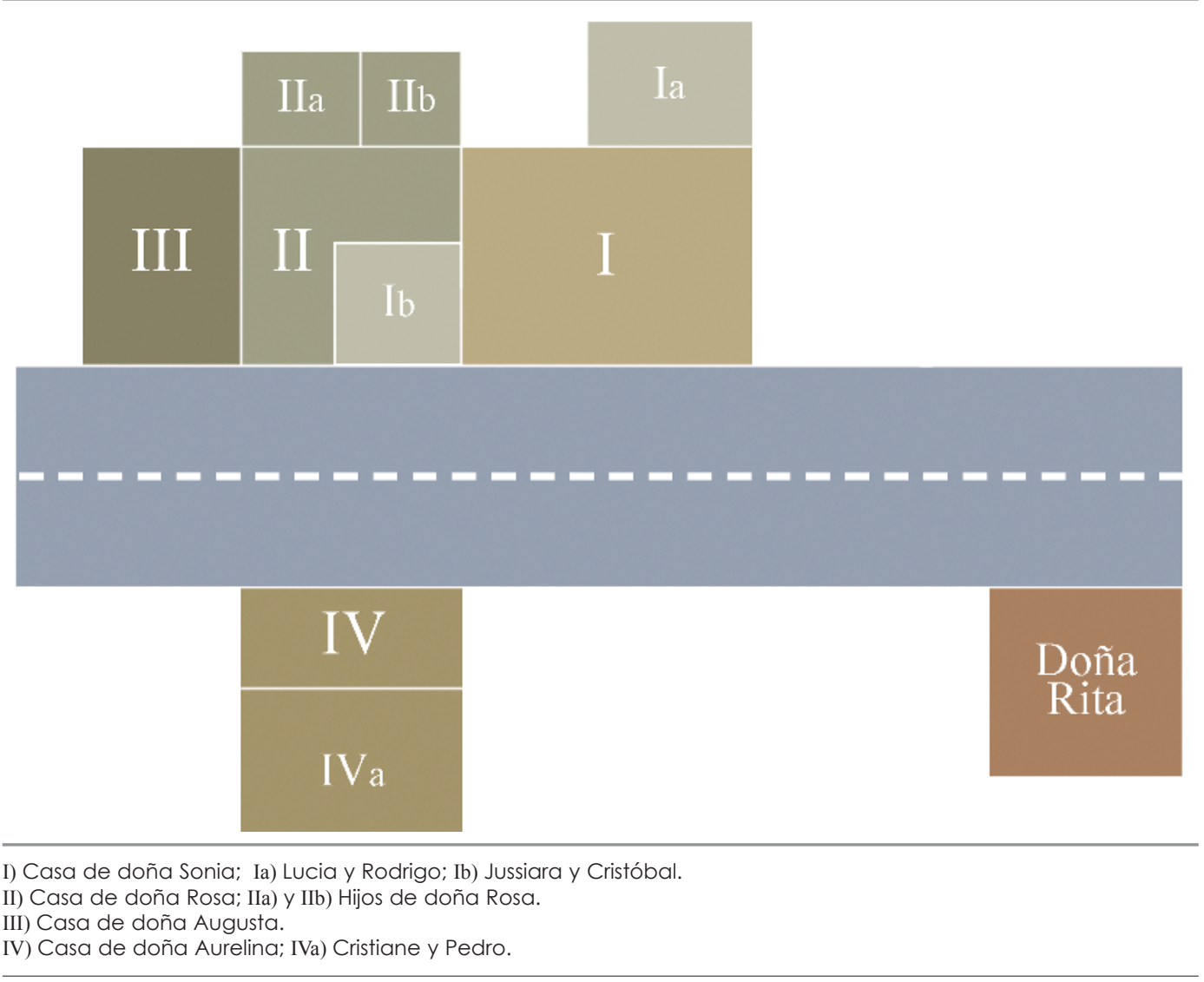

Fuente: elaboración propia. 
casas Ia y Ib realizan actividades vinculadas con la supervivencia en la casa I -preparación de alimentos e higiene-, y eventualmente lo hacen en la casa II, pero sería raro que realicen estas actividades en las otras casas.

Las relaciones entre miembros de diferentes subconjuntos se expresan en intercambios de alimentos y otros objetos. Un ejemplo cotidiano era la comida de los niños de una casa, que podía ser completada por miembros de otra casa. La ayuda en momentos especiales también era común, como cuando doña Rosa necesitaba asistencia médica. Entonces, era doña Sonia, y no alguno de los hijos de doña Rosa, quien la llevaba a las consultas médicas o a los servicios de emergencia.

Otra expresión de la "conectividad" que existe entre estas personas es que están al tanto de la vida de los otros. Cuando la investigadora llegaba a la casa de doña Aurelina alguien podía contar algo sobre doña Sonia y sus hijos -por ejemplo, "Lucia está embarazada" o "Jussiara se fue a la iglesia"- y lo mismo podría darse en la casa de doña Sonia: "Cristiane tiene un dolor de muelas muy fuerte", me advirtieron un día Jussiara y Cristóbal.

\section{Sangre, consideración y redes}

Como en la investigación de Marcelin (21), también en Prainha, "sangre" y "consideración" son principios para construir el parentesco, pero ninguno de ellos es suficiente por sí solo. Para el citado autor, "existe una concepción de familia y de pariente fundamentada en los principios de la bilateralidad de la herencia de la sangre y de la consideración" (traducción nuestra) (21). El principio de sangre remite a una sustancia común, compartida entre individuos que tienen los mismos padres. La bilateralidad proporciona la posibilidad de construir la distinción entre el lado materno y el paterno. Sin embargo, el uso de la familia y del parentesco es selectivo. "Los agentes seleccionan a sus parientes, o se aproximan a ellos de acuerdo con sus intereses específicos. El reconocimiento efectivo coloca en acción mecanismos de selección: la consideración es la selección en acción" (traducción nuestra) (21). En Prainha, la "consideración" toma las formas de las categorías de parentesco convencionales -por ejemplo, madre, tía, abuela o madrina de "consideración"- construidas a partir de intercambios, en los que queda claro que existen afecto y afinidad y que estos se expresan en la posibilidad de recibir y dar ayuda.

Existen parientes de sangre que "no se llevan bien", como Diogo y sus hermanas que viven en la planta baja. Los vínculos consanguíneos son identificados y envuelven cierto respeto, como cuando doña Sonia dice, criticando a uno de sus hijos: "Vive tomando cachaça (c), yo lo dejo entrar porque es mi hijo, pero él no vale nada", y se consolidan cuando existe "consideración". La propia doña Sonia tiene una abierta preferencia por su hijo Rodrigo, que vive cerca de ella y es el que más cubre sus expectativas.

Las relaciones de pareja son apenas una de las dimensiones de la construcción de los vínculos familiares. Aquí analizamos el caso de las hermanas Alicia y Lucineide en cuyo cotidiano las relaciones con parientes consanguíneos (madre, hermanos, sobrinos, entre otros) y de "consideración", son centrales. Para ambas el carácter temporal de las relaciones de pareja contrasta con las relaciones permanentes de los parentescos consanguíneos, y esto se expresa en la propiedad de la casa, que es el mayor bien material que poseen. Ambas construyeron sus casas sin ayuda de sus parejas, utilizando la azotea que fue brindada por la madre.

Es parte del cotidiano de las dos hermanas y de sus hijos el tránsito por varias casas, especialmente en el conjunto de las tres casas más próximas: las de Lucineide, Alicia y doña Neda. Los intercambios entre los miembros de las tres casas abarcan aspectos cotidianos como la alimentación y el contacto con servicios de salud. A veces, cuando Alicia necesita llevar a su hijo Anderson a un servicio de emergencia por alguna crisis de asma, Lucineide y la madre de Alicia cuidan a los otros hijos y algunas veces, la hija menor se queda en casa de la abuela materna. En esas ocasiones, la presencia de los hermanos se hace más visible. Marcio le presta dinero y facilita el acceso a los servicios de la unidad de salud familiar en la que trabaja como agente comunitario, o Manuel la acompaña aliviándola del costo del traslado, por tener derecho al uso libre del transporte público para él y para un acompañante. 
Con relación a una investigación realizada en la ciudad de Porto Alegre, Fonseca (1) notó con frecuencia una gran complicidad y escasas tensiones entre hermanos y hermanas, a diferencia de lo que sucedía en las relaciones entre cónyuges o entre parientes mujeres. En Prainha, tal como ilustra el caso de Alicia, Marcio y Manuel, existe una significativa cooperación entre los hermanos, especialmente en momentos de crisis.

Al comparar diferentes relaciones de parentesco vemos que la consanguinidad es privilegiada, tal como sugieren los análisis de Fonseca (1). Doña Aurelina declara que "considera" como nieto a Alex, hijo de la primera unión de Cristiane. Ambas mujeres comparten su vida cotidiana. Cristiane tiene su casa, pero pasa la mayor parte del tiempo en la casa de la suegra, porque las dos "se llevan muy bien". Sin embargo, cuando surgió la posibilidad de un trabajo, doña Aurelina no permitió que Cristiane lo aceptara porque ella no podía cuidar todos los días a Tadeo (hijo de Cristiane de dos años). Doña Aurelina dijo que era mejor que su hija Carmen -que vivía en otro barrio y había dejado a su hijo con ella- se quedara con el trabajo.

Las relaciones de parentesco de consideración no excluyen problemas de comunicación ni críticas. Si bien doña Sonia "considera" a doña Aurelina y sus hijos como parientes, ella critica la opción de su amiga por la religión Testigos de Jehová. En otra ocasión doña Sonia habló mal de la familia de doña Rosa, la madre de su yerno Cristóbal: "En esa familia todos son ladrones, el hermano cogió un arma y ahora los bandidos lo quieren matar si no paga 400 reales, todos los hermanos están desesperados tratando de conseguir esos 400".

Los vínculos de consideración tampoco están garantizados. Un ejemplo de eso es la relación de Cristiane -la nuera de doña Aurelina, que vive en el barrio hace poco tiempo- con los vecinos de la misma calle, especialmente los "vecinos-familia". En algunas ocasiones escuché a doña Sonia, doña Rosa, Jussiara y una hija de doña Rosa criticando el comportamiento de Cristiane: "Ella no se lleva bien con nadie de esta calle, es una chismosa, tramposa".

La relación con doña Rita (Figura 2) muestra cómo la proximidad física y los parientes en común no son motivos suficientes para construir relaciones de parentesco. Es necesario que existan afinidades. Cuando doña Sonia vio que yo estaba visitando la casa de doña Rita, me dijo: "Hace mucho tiempo que conozco a Rita. Su hija tuvo un bebé con mi hijo. Pero a ella le gusta la macumba (d) y eso no es bueno. Si no, mira como es su casa."

El cuidado frente a problemas de salud es una buena oportunidad para la construcción de relaciones de parentesco. Cuando la pequeña Sandra se enfermó y tuvo que ser hospitalizada por algunos días, sus padres Paula y Ed -él trabajaba por la noche- no tenían ayuda. Luana, la novia de un amigo de Ed, se enteró de la situación, visitó a Sandra e insistió en quedarse en el hospital por las noches. Eso fue muy importante para la pareja, que después le pidió a Luana que fuera la madrina de Sandra. Así, es común que existan madrinas y padrinos y, generalmente, esta relación no implica una ceremonia religiosa, sino un vínculo de consideración.

A diferencia del análisis de Marcelin (21), en este estudio identificamos dos niveles en la organización de la configuración de casas, que pueden ser relacionados con los principios de

Figura 2. Las casas de doña Sonia y sus hijos.

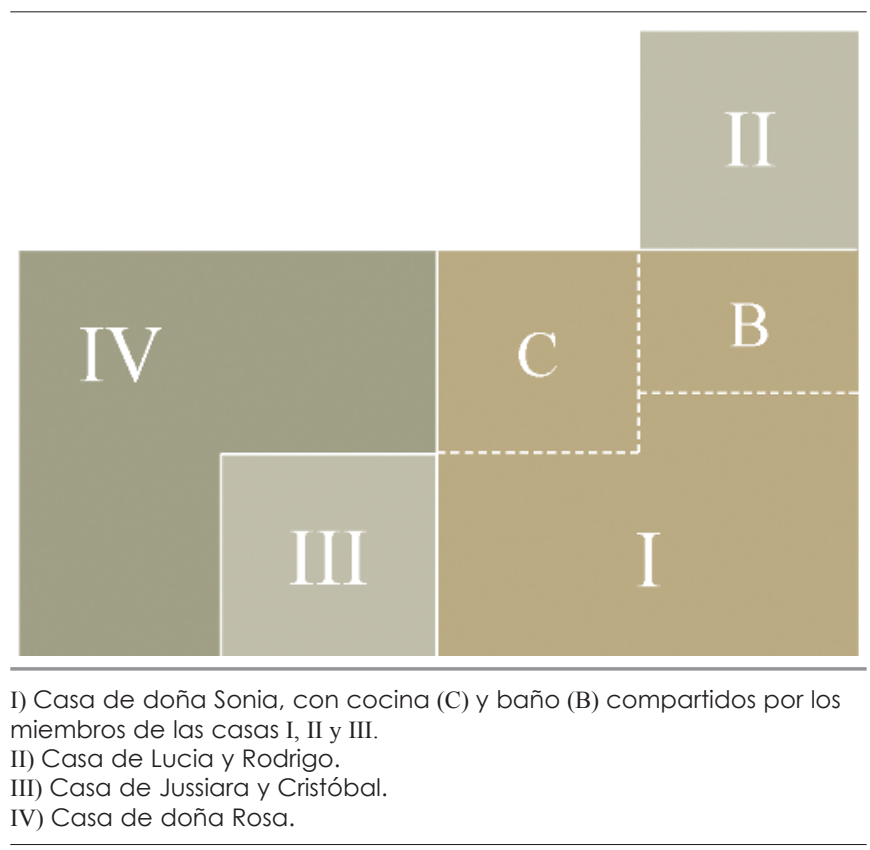

Fuente: elaboración propia. 
"sangre" y "consideración". Existe una configuración de casas formada por las casas de parientes próximos -por ejemplo, las casas de doña Sonia y sus hijos- que son parientes de "sangre". Por otro lado, existe una configuración más amplia integrada por "vecinos-familia" con quienes existen lazos de consideración. Esto puede tener importantes consecuencias para pensar la organización de cuidados en salud. Los parientes de sangre son los primeros, pero no los únicos que asumen responsabilidades por el cuidado de la salud de niños y ancianos. Después de los parientes de sangre y de consideración cabe a los "vecinos-familia" ayudar en los cuidados de salud. Por otro lado, aquí mostramos que la ayuda frente a problemas de salud es un momento privilegiado para la construcción de lazos de consideración, que no necesariamente se refieren a personas que integran la configuración de casas.

\section{CONCLUSIÓN}

En este estudio buscamos identificar algunos aspectos importantes en la literatura sobre la familia en el contexto del PSF: describimos cómo se aborda el tema "familia" en documentos oficiales del PSF; mencionamos investigaciones sobre la comprensión que tienen los profesionales del equipo acerca de las familias atendidas en ese programa, así como estudios sobre los cuidados de la salud en el contexto familiar. Llamamos la atención sobre la noción de familia que predomina en estos documentos e investigaciones: el énfasis en la conyugalidad y la co-residencia como criterios para definir familia. En contraposición mostramos datos etnográficos que evidencian la gran importancia de la relación entre los miembros de las casas y la configuración de las casas y la construcción de lazos de "sangre" y "consideración".

Este estudio tiene como limitación no incluir directamente la perspectiva de los profesionales de la salud. Esta es una tarea que deberá ser realizada en una próxima investigación. Sin embargo, con base en la revisión de la literatura, es posible hacer algunas reflexiones sobre las prácticas al interior del PSF con relación a las familias.
Así, para pensar cómo las personas organizan su vida cotidiana, incluyendo los cuidados de la salud, no se trata solo de analizar la relación entre familias y redes sociales. Entender la relación entre las casas y la configuración de casas, así como los lazos de "sangre" y "consideración", puede contribuir a construir intervenciones profesionales más efectivas, en la medida en que se identifiquen los vínculos que pueden ser accionados frente a necesidades y problemas de salud.

Esta reflexión se suma a las sugerencias de Muniz y Eisenstein (28) sobre la necesidad de incluir el uso del genograma como complemento de la anamnesis tradicional. Los autores consideran que este instrumento favorece la identificación de estresores en el contexto familiar y su influencia en el proceso salud-enfermedad $y$ también permite identificar patrones transgeneracionales de enfermedades y de redes de apoyo psicosocial, además de posibilitar la ampliación de estrategias terapéuticas más adecuadas. A diferencia de los autores citados, defendemos que en lugar de usar instrumentos estandarizados es fundamental que los profesionales estén atentos a entender las dinámicas de parentesco que se organizan en cada territorio. Existen criterios para organizar las relaciones de parentesco que, inicialmente, son difíciles de identificar. Los profesionales pueden mejorar su aproximación usando las referencias a "sangre" y "consideración" como lenguajes que están presentes y que pueden ser fortalecidos. Al mismo tiempo es importante no partir de idealizaciones sobre la vida en los barrios de bajos ingresos. En ese sentido, aquí mostramos que las relaciones de parentesco implican reciprocidad y requieren ser construidas en la vida cotidiana para que puedan ser efectivas.

Al ampliar el enfoque hacia la relación entre casa y configuración de casas y lazos de sangre y consideración podemos ver otros aspectos de la presencia masculina que no se limitan a la conyugalidad. Los hombres aparecieron en este estudio como muy importantes en su condición de hijos, hermanos o tíos. Esta mirada sobre la participación masculina podría ser útil para estimular la inclusión de los hombres en las prácticas y cuidados de salud.

Otro aspecto importante, que contrasta con la forma en que la familia es pensada en los 
documentos que formulan el PSF, es la relación entre casa y familia. Mientras que en los documentos se recomienda registrar como familia a un grupo de personas que conviven en una casa, aquí mostramos que el concepto de casa es bastante complejo -un espacio puede ser apenas una habitación para una mirada externa mientras que para quien lo habita puede ser una casa- y que la casa existe necesariamente dentro de una configuración de casas, algo que no está contemplado en los registros sobre las familias atendidas por un equipo de salud.

El desafío para los profesionales es todavía mayor si recordamos que, entre los informantes, el modelo familiar nuclear $-y$ la mirada sobre la familia a partir de la conyugalidad- está muy presente, principalmente, en el plano discursivo. Aquí mostramos que existen relaciones flexibles entre el modelo valorizado y la organización cotidiana de las prácticas. Así, entre los habitantes de Prainha, la valoración de modelos que priorizan a la familia nuclear y la conyugalidad -tal como está en los documentos del PSF y en algunas investigaciones sobre familia- coexiste con la enorme creatividad de la construcción de la vida cotidiana. Todo lo descrito se refiere a una comprensión del parentesco como proceso en permanente construcción.

\section{AGRADECIMIENTOS}

Vania Bustamante recibió becas de doctorado del Conselho Nacional de Desenvolvimento Científico e Tecnológico $(\mathrm{CNPq})$ y de posdoctorado de la Fundação de Apóio à Pesquisa do Estado da Bahia (FAPESB).

\section{NOTAS FINALES}
a. El término prainha significa "playita".
b. Pequeño municipio del interior de Bahía.

c. Es un destilado de caña de azúcar muy consumido en todo Brasil.

d. Es una forma un tanto despectiva de referirse al Candomblé, una religión de origen africano que está bastante presente en Salvador y otras regiones.

\section{REFERENCIAS BIBLIOGRÁFICAS}

1. Fonseca C. Família, fofoca e a honra. Etnografia de relações de gênero y violência em grupos populares. 2a ed. Porto Alegre: UFRGS; 2003.

2. Brasil. Ministério da Saúde. Pré-natal e puerpério: atenção qualificada e humanizada (Manual técnico). Brasília: Ministério da Saúde; 2005.

3. Brasil. Ministério da Saúde. Guia prático do Programa de Saúde da família. Brasília: Ministério da Saúde; 2002.

4. Brasil. Ministério da Saúde. Saúde da família: una estratégia para a reorganização do modelo assistencial. Brasília: Ministério da Saúde; 1997.

5. Brasil. Ministério da Saúde. Atenção Básica e a Saúde da Família [Internet] Brasília: Departamento de Atenção Básica [citado 19 may 2011]. Disponible en: http://dab.saude.gov.br/ abnumeros.php
6. Vasconcelos FGA, Zaniboni MRG. Dificuldades do trabalho médico no PSF. Ciência e Saúde Coletiva. 2011;16(supl.1):S1497-S1504.

7. Serapioni M. O papel da família e das redes sociais na reestruturação das políticas sociais. Ciência e Saúde Coletiva. 2005;10(supl):S243S253.

8. Ribeiro EM. As várias abordagens da família no cenário do Programa/Estratégia de Saúde da família (PSF). Revista Latino-Americana de Enfermagem. 2004; 12:658-664.

9. Resta DG, Motta MGC. Família em situação de risco e sua inserção no Programa de Saúde da família: una reflexão necessária à prática profissional. Texto \& Contexto Enfermagem. 2005;14:109-115.

10. Gabardo RM, Jungues JR, Selli L. Arranjos familiares e implicações à saúde na visão do Programa Saúde da família. Revista de Saúde Pública. 2009;43:91-97. 
11. Yunes MAM, Mendes NF, Albuquerque BM. Percepções e crenças de agentes comunitários de saúde sobre resiliência em famílias monoparentais pobres. Texto \& Contexto Enfermagem. 2005; 14:24-31.

12. Sarti CA. Família e individualidade: um problema moderno. En: Carvalho MCB, organizadora. A família Contemporânea em Debate. São Paulo: EDUC; 1994. p. 39-49.

13. Heilborn ML. O traçado da vida: gênero e idade em dois bairros populares do Rio de Janeiro. En: Reicher Madeira F, organizadora. Quem mandou nascer mulher? Estudos sobre crianças e adolescentes pobres no Brasil. Rio de Janeiro: Record/Rosa dos Tempos; 1997. p. 292-339.

14. Szymanski H. Teorias e "teorias" de famílias. En: Carvalho MCB, organizadora. A família Contemporânea em Debate. São Paulo: EDUC; 1995. p.23-28.

15. Fonseca C. "Mãe é uma só?". Reflexões em torno de alguns casos brasileiros. Revista de Psicologia USP. 2002;13:49-68.

16. Velho G. Individualismo e cultura. Notas para una antropologia da sociedade contemporânea. Rio de Janeiro: Zahar; 1981.

17. Woortmann K. A família das mulheres. Rio de Janeiro: Tempo Brasileiro; 1987.

18. Agier M. O sexo da pobreza. Homens, Mulheres e famílias numa "Avenida" em Salvador da Bahia. Tempo Social. 1990;2(2):35-60.

19. Agier M. Espaço urbano, família e status social: o novo operariado baiano nos seus bairros. Cadernos do Centro de Recursos Humanos. 1990;13:39-62.
20. Hita MG. Pobreza, composición familiar e inclusión social: arreglo matriarcal en un Brasil negro. En: Anales del Seminario Internacional "Pobreza, Exclusión y discriminación ÉtnicoRacial en América Latina y el Caribe". Cali: Clacso-Crop; 2006.

21. Marcelin LH. A linguagem da casa entre os negros do Recôncavo Baiano. Mana. 1999;5:31-60.

22. Carsten J. After kinship. Cambridge: Cambridge University Press; 2004.

23. Bustamante V. Cuidado infantil e construção social da pessoa: uma etnografia em um bairro popular de Salvador. [Tesis de doctorado]. Salvador: Universidade Federal da Bahia: 2009.

24. Jackson $M$, editor. Things as they are. New directions in phenomenological anthropology. Bloomington: Indiana University Press; 1996.

25. Toren C. Ethnography: theoretical background. En: Richardon JTE, editor. Handbook of qualitative research methods for psychology and the Social Sciences. London: BPS; 1997. p.102112.

26. Becker H. Métodos de pesquisa em Ciências Sociais. São Paulo: HUCITEC; 1994.

27. Pina-Cabral JO. Homem na família: cinco ensaios de antropologia. Lisboa: Imprensa de Ciências Sociais; 2003.

28. Muniz JR, Eisenstein E. Genograma: informações sobre família na (in)formação médica. Revista Brasileira de Educação Médica. 2009;33:72-79.

\section{FORMA DE CITAR}

Bustamante V, McCallum CA. Parentesco y casas en un barrio de bajos ingresos asistido por el Programa de Salud Familiar en Salvador, Bahía, Brasil. Salud Colectiva. 2011;7(3):365-376.

Recibido el 18 de noviembre de 2010

Versión final presentada el 16 de mayo de 2011

Aprobado el 17 de junio de 2011 